\title{
NONOSCILLATION OF THE SOLUTIONS OF IMPULSIVE DIFFERENTIAL EQUATIONS OF THIRD ORDER
}

\author{
MARGARITA BONEVA DIMITROVA \\ Technical University \\ Department of Mathematics \\ Sliven 8800, Bulgaria
}

(Received June, 1998; Revised November, 1999)

\begin{abstract}
Necessary and sufficient conditions are found for existence of at least one bounded nonoscillatory solution of a class of impulsive differential equations of third order and fixed moments of impulse effect. Some asymptotic properties of the nonoscillating solutions are investigated.
\end{abstract}

Key words: Oscillation, Impulsive Differential Equations.

AMS subject classifications: $34 \mathrm{~A} 37$.

\section{Introduction}

The impulsive differential equations with deviating argument are adequate mathematical models of numerous processes and phenomena in physics, biology and electrical engineering. In spite of wide possibilities for their application, the theory of these equations is developing rather slowly because of considerable difficulties in technical and theoretical character related to their study.

In the recent twenty years, the number of investigations devoted to the oscillatory and nonoscillatory behavior of the solutions of functional differential equations has considerably increased. A large part of the works of this subject published in 1977 is presented in [5]. In monographs [2] and [3], published in 1987 and 1991, respectively, the oscillatory and asymptotic properties of the solutions of various classes of functional differential equations were systematically studied. A pioneering work devoted to the investigation of the oscillatory properties of the solutions of impulsive differential equations with deviating argument was rendered by Gopalsamy and Zhang [1].

In the present paper, necessary and sufficient conditions are found for existence of at least one bounded nonoscillatory solution of a class of impulsive differential equations of third order and fixed moments of impulse effect. Some asymptotic properties of the nonoscillating solutions are investigated. 


\section{Preliminary Notes}

Consider the impulsive differential equation

$$
\begin{gathered}
y^{\prime \prime \prime}(t)+f(t, y(t))=0, \quad t \neq \tau_{k}, k \in N, \\
\Delta y^{\prime \prime}\left(\tau_{k}\right)+f_{k}\left(y\left(\tau_{k}\right)\right)=0, \quad k \in N, \\
\Delta y^{\prime}\left(\tau_{k}\right)=\Delta y\left(\tau_{k}\right)=0, \quad k \in N
\end{gathered}
$$

with initial conditions

where $y_{i} \in R, i=0,1,2$.

$$
y^{(i)}(0)=y_{i}
$$

Here $\Delta y^{\prime \prime}\left(\tau_{k}\right)=y^{\prime \prime}\left(\tau_{k}+0\right)-y^{\prime \prime}\left(\tau_{k}-0\right)$. We suppose that $y\left(\tau_{k}-0\right)=y\left(\tau_{k}\right)$; $y^{\prime}\left(\tau_{k}-0\right)=y^{\prime}\left(\tau_{k}\right) ; R_{+}=(0,+\infty) ; \tau_{1}, \tau_{2}, \ldots$ are the moments of impulse effect.

Introduce the following conditions:

H1. $0<\tau_{1}<\tau_{2}<\ldots, \lim _{k \rightarrow+\infty} \tau_{k}=+\infty$.

H2. $f \in C\left(R_{+} \times R, R\right), u f(t, u)>0$ for $u \neq 0, t \in R_{+} ;\left|f\left(t, u_{1}\right)\right| \leq\left|f\left(t, u_{2}\right)\right|$ for $\left|u_{1}\right| \leq\left|u_{2}\right|, u_{1}, u_{2} \in R, t \in R_{+}$.

H3. $f_{k} \in C(R, R), u f_{k}(u)>0$ for $u \neq 0$ and $\left|f_{k}\left(u_{1}\right)\right| \leq\left|f_{k}\left(u_{2}\right)\right|$ for $\left|u_{1}\right| \leq\left|u_{2}\right|, u_{1}, u_{2} \in R, k \in N$.

Definition 1: A function $y \in C\left(R_{+}, R\right)$ is called a solution of the equation (1) with initial conditions (2) if it satisfies the following conditions:

(a) If $0=\tau_{0} \leq t \leq \tau_{1}$, then the function $y$ coincides with the solution of the equation

$$
y^{\prime \prime \prime}(t)+f(t, y(t))=0
$$

with initial conditions (2).

(b) If $\tau_{k}<t \leq \tau_{k+1}$, then the function $y$ coincides with the solution of the equation

with initial conditions

$$
y^{\prime \prime \prime}(t)+f(t, y(t))=0
$$

$$
\begin{gathered}
y^{\prime \prime}\left(\tau_{k}+0\right)=y^{\prime \prime}\left(\tau_{k}-0\right)-f_{k}\left(y\left(\tau_{k}\right)\right), \\
y^{\prime}\left(\tau_{k}+0\right)=y^{\prime}\left(\tau_{k}-0\right)=y^{\prime}\left(\tau_{k}\right), \\
y\left(\tau_{k}+0\right)=y\left(\tau_{k}-0\right)=y\left(\tau_{k}\right) .
\end{gathered}
$$

Definition 2: The nonzero solution $y(t)$ of the problem (1), (2) is said to be nonoscillatory if there exists a point $t_{0} \geq 0$ such that $y(t)$ has a constant sign for $t \geq t_{0}$. Otherwise, the solution $y(t)$ is said to oscillate.

Definition 3: ([4]) A set $\Omega$ of real-valued functions defined on the interval $\left[t_{0},+\infty\right)$ is said to be equiconvergent at $\infty$ if all functions in $\Omega$ are convergent in $R$ as $t \rightarrow \infty$ and for any $\varepsilon>0$ there exists $t_{0}^{\prime} \geq t_{0}$ such that for each function $f \in \Omega$, the inequality $\left|f(t)-\lim _{s \rightarrow \infty} f(s)\right|<\varepsilon$ is valid for $t \geq t_{0}^{\prime}$.

Lemma 1: ([4]) Let $\Omega$ be uniformly bounded and an equicontinuous subset of the Banach space $B\left(\left[t_{0}, \infty\right)\right)$, and let $\Omega$ be equiconvergent at $\infty$. then the set $\Omega$ is relatively compact. 


\section{Main Results}

Theorem 1: Let the following conditions hold:

(a) Conditions $\boldsymbol{H 1 - H 3}$ are met.

(b) There exists a point $T \geq 0$ such that

$$
\int_{T}^{\infty}(u-T)^{2}|f(u, c)| d u+\sum_{T<\tau_{k}}\left(\tau_{k}-T\right)^{2}\left|f_{k}(c)\right|=+\infty
$$

for some constant $c \neq 0$.

Then every bounded solution $y(t)$ of the equation (1) either oscillates or

$$
\lim _{t \rightarrow+\infty} y(t)=\lim _{t \rightarrow+\infty} y^{\prime}(t)=\lim _{t \rightarrow+\infty} y^{\prime \prime}(t)=0 .
$$

Proof: Let $y(t)$ be a positive and bounded solution of the equation (1) for $t \geq t_{1} \geq 0$. From condition $\mathbf{H 3}, f_{k}\left(y\left(\tau_{k}\right)\right)>0$ for $\tau_{k} \geq t_{1}$. Then

$$
\Delta y^{\prime \prime}\left(\tau_{k}\right)<0, \quad \tau_{k} \geq t_{1} \text {. }
$$

From $y(t)>0, t \geq t_{1}$ and condition $\boldsymbol{H} 2$ implies $f(t, y(t))>0, t>t_{1}$. Therefore,

$$
y^{\prime \prime \prime}(t)<0, \quad t \geq t_{1}
$$

From (3) and (4) it follows that $y^{\prime \prime}(t)$ is a decreasing function for $t \geq t_{1}$.

The following two cases are possible:

Case 1: $y^{\prime \prime}(t)>0$ for $t \geq t_{1}$. Then $y^{\prime}(t)$ is an increasing function for $t \geq t_{1}$.

1.1: If $y^{\prime}(t)>0$ for $t \geq t_{2} \geq t_{1}$, then $y^{\prime}(t) \geq y^{\prime}\left(t_{2}\right)>0$. We integrate the last inequality from $t_{2}$ to $t\left(t \geq t_{2}\right)$ and conclude

$$
y(t) \geq y^{\prime}\left(t_{2}\right)\left(t-t_{2}\right)+y\left(t_{2}\right)
$$

It follows from the above inequality as $t \rightarrow+\infty$, that $\lim _{t \rightarrow \infty} y(t)=+\infty$ which contradicts the assumption that $y$ is a bounded solution of the equation (1).

1.2: If $y^{\prime}(t)<0$ for $t \geq t_{2} \geq t_{1}$. Then $y(t)$ is decreasing and bounded, so there exists a limit, $\lim _{t \rightarrow+\infty} y(t)=c_{1} \geq 0$. From $y^{\prime \prime}(t)>0, \Delta y^{\prime}\left(\tau_{k}\right)=0$ for $t, \tau_{k} \geq t_{1}$ to see that $y^{\prime}(t)$ is an increasing negative function. Therefore, $\lim _{t \rightarrow+\infty} y^{\prime}(t)=c_{2} \leq 0$.

Let us suppose $c_{2}<0$. Then there exists a constant $c_{3}<0$ and a point $t_{3} \geq t_{2}$ such that $y^{\prime}(t) \leq c_{3}$ for $t \geq t_{3}$. Now, we integrate the above inequality from $t_{3}$ to $t$, $\left(t \geq t_{3}\right)$ and arrive at the inequality $y(t) \leq c_{3} t+y\left(t_{3}\right)$. It follows from the above inequality after taking the limit as $t \rightarrow+\infty$, that $\lim _{t \rightarrow+\infty} y(t)=-\infty$, which contradicts the assumption that $y$ is a positive bounded solution of the equation (1). Therefore,

$$
\lim _{t \rightarrow+\infty} y^{\prime}(t)=0
$$

From $y^{\prime \prime \prime}(t)<0, \Delta y^{\prime \prime}\left(\tau_{k}\right)<0$ for $t, \tau_{k} \geq t_{1}$ we see that $y^{\prime \prime}(t)$ is a decreasing positive function. Therefore, $\lim _{t \rightarrow+\infty} y^{\prime \prime}(t)=c_{4} \geq 0$. We want to prove that $c_{4}=0$. Assume that $c_{4}>0$. Then there exists a constant $c_{5}>0$ and a point $t_{4} \geq t_{1}$ such that $y^{\prime \prime}(t) \geq c_{5}$ for $t \geq t_{4}$. Now, we integrate the above inequality from $t_{4}$ to $t$, 
$\left(t \geq t_{4}\right)$ and arrive at the inequality

$$
y^{\prime}(t) \geq c_{5}\left(t-t_{4}\right)+y^{\prime}\left(t_{4}\right)
$$

It follows from (5) and after taking the limit as $t \rightarrow+\infty$, that $\lim _{t \rightarrow+\infty} y^{\prime}(t)=$ $+\infty$, which contradicts that $\lim _{t \rightarrow+\infty} y^{\prime}(t)=0$. Therefore,

$$
\lim _{t \rightarrow+\infty} y^{\prime \prime}(t)=0 \text {. }
$$

Let us suppose $\lim _{t \rightarrow+\infty} y(t)=c_{1}>0$. But $y$ is a bounded, continuous, decreasing and positive function. Thus, there exists constants $c>0, c_{6}>0$ and point $t_{5} \geq t_{1}$ such that $c \leq y(t) \leq c_{6}$ for $t \geq t_{5}$.

We integrate (1) from $t$ to $+\infty,\left(t \geq t_{5}\right)$ and arrive at the equality

$$
\lim _{t \rightarrow+\infty} y^{\prime \prime}(t)-y^{\prime \prime}(t)-\sum_{\tau_{k} \geq t} \Delta y^{\prime \prime}\left(\tau_{k}\right)+\int_{t}^{\infty} f(u, y(u)) d u=0 .
$$

From $y(t) \geq c$ for $t \geq t_{5}$ and conditions $\mathbf{H} 2$ and H3, we have

$$
y^{\prime \prime}(t) \geq \int_{t}^{\infty} f(u, c) d u+\sum_{\tau_{k} \geq t} f_{k}(c)
$$

We integrate the last inequality from $s$ to $\infty$ and conclude

$$
\begin{gathered}
\lim _{t \rightarrow+\infty} y^{\prime}(t)-y^{\prime}(s) \geq \int_{s}^{\infty}\left[\int_{t}^{\infty} f(u, c) d u+\sum_{\tau_{k} \geq t} f_{k}(c)\right] d t \\
=\int_{t}^{\infty}(u-t) f(u, c) d u+\sum_{\tau_{k} \geq t}\left(\tau_{k}-t\right) f_{k}(c)
\end{gathered}
$$

i.e.

$$
-y^{\prime}(t) \geq \int_{t}^{\infty}(u-t) f(u, c) d u+\sum_{\tau_{k} \geq t}\left(\tau_{k}-t\right) f_{k}(c) \text {. }
$$

Integrating (6) from $T$ to $t,(t \geq T)$, we obtain

$$
y(T)-y(t) \geq \int_{T}^{t}\left[\int_{s}^{\infty}(u-s) f(u, c) d u+\sum_{\tau_{k} \geq s}\left(\tau_{k}-s\right) f_{k}(c)\right] d s,
$$

or

$$
\begin{gathered}
y(T)-y(t) \geq \frac{1}{2} \int_{T}^{t}(u-T)^{2} f(u, c) d u+\frac{1}{2} \sum_{T \leq \tau_{k} \leq t}\left(\tau_{k}-T\right)^{2} f_{k}(c) d s \\
-\lim _{t \rightarrow+\infty} y(t) \geq \frac{1}{2} \int_{T}^{\infty}(u-T)^{2} f(u, c) d u+\frac{1}{2} \sum_{T \leq \tau_{k}}\left(\tau_{k}-T\right)^{2} f_{k}(c) .
\end{gathered}
$$


The last inequality contradicts condition 2 of Theorem 1 . Therefore,

$$
\lim _{t \rightarrow+\infty} y(t)=0 \text {. }
$$

Case 2: $y^{\prime \prime}(t)<0$ for $t \geq t_{1}$.

It is easy to see that $\lim _{t \rightarrow+\infty} y(t)=-\infty$. This contradicts the assumption $y(t)>0$. The proof is complete.

Theorem 2: Let the following conditions hold:

(1) Conditions H1-H3 are met.

(2)

$$
\int^{\infty} t^{2}|f(t, c)| d t+\sum_{k=1}^{\infty} \tau_{k}^{2}\left|f_{k}(c)\right|<+\infty
$$

for some constant $c \neq 0$.

Then the equation (1) has a bounded nonoscillatory solution.

Proof: From condition (2), one can find a sufficiently large $T \geq 0$ such that

$$
\int_{T}^{\infty} t^{2}|f(u, c)| d t+\sum_{\tau \geq T} \tau_{k}^{2}\left|f_{k}(c)\right| \leq|c|
$$

Let $X$ be the space of bounded continuous functions on $[T, \infty)$. Let $Y \subset X$ be defined by

$$
Y=\left\{y \in X ; \frac{|c|}{2} \leq y \quad \operatorname{sign} c \leq|c|\right\}
$$

Then $Y$ is a bounded convex closed subset of $X$.

Define the operator $S: Y \rightarrow X$ as follows:

$(S y)(t)=\left\{\begin{array}{c}\frac{c}{2}+\frac{1}{2} \int_{t}^{\infty}(s-t)^{2} f(s, y(s)) d s+\frac{1}{2} \sum_{\tau_{k} \geq t}\left(\tau_{k}-t\right)^{2} f_{k}\left(y\left(\tau_{k}\right)\right), \quad t \geq T \\ \frac{c}{2}+\frac{1}{2} \int_{T}^{\infty}(s-T)^{2} f\left(s, y(s) d s+\frac{1}{2} \sum_{\tau_{k} \geq T}\left(\tau_{k}-T\right)^{2} f_{k}\left(y\left(\tau_{k}\right)\right) \quad 0 \leq t \leq T .\right.\end{array}\right.$

(a) $S$ maps $Y$ into itself. In fact

$$
\begin{aligned}
\frac{|c|}{2} & \leq(S y)(t) \operatorname{sign} c \leq \frac{|c|}{2}+\frac{1}{2} \int_{t}^{\infty}(s-t)^{2}|f(s, c)| d s+\frac{1}{2} \sum_{\tau_{k} \geq t}\left(\tau_{k}-t\right)^{2}\left|f_{k}(c)\right| \\
& \leq \frac{|c|}{2}+\frac{1}{2} \int_{t}^{\infty} s^{2}|f(s, c)| d s+\frac{1}{2} \sum_{\tau_{k} \geq t} \tau_{k}^{2}\left|f_{k}(c)\right| \leq \frac{|c|}{2}+\frac{|c|}{2}=|c|,
\end{aligned}
$$

due to conditions H2, H3 and (7).

(b) $S$ is continuous. To prove this, let $\left\{y_{n}\right\}$ be a Cauchy sequence in $Y$, and let $\lim _{t \rightarrow+\infty}\left\|y_{n}-y\right\|=0$. Because $Y$ is closed, $y \in Y$. To prove the continuity of $S$, we see that

$$
\left|\left(S y_{n}\right)(t)-(S y)(t)\right|
$$


$\leq \frac{1}{2} \int_{t}^{\infty}(s-t)^{2}\left|f\left(s, y_{n}(s)\right)-f(s, y(s))\right| d s+\frac{1}{2} \sum_{\tau_{k} \geq t}\left(\tau_{k}-t\right)^{2}\left|f_{k}\left(y_{n}\left(t_{k}\right)\right)-f_{k}\left(y\left(\tau_{k}\right)\right)\right|$

Set

$$
\begin{gathered}
F_{n}(s)=s^{2}\left|f\left(s, y_{n}(s)\right)-f(s, y(s))\right| \\
L_{n}\left(\tau_{k}\right)=\tau_{k}^{2}\left|f_{k}\left(y_{n}\left(\tau_{k}\right)\right)-f_{k}\left(y\left(\tau_{k}\right)\right)\right| .
\end{gathered}
$$

Then (8) reduces to

$$
\left|\left(S y_{n}\right)(t)-(S y)(t)\right| \leq \frac{1}{2} \int_{t}^{\infty} F_{n}(s) d s+\frac{1}{2} \sum_{\tau_{k} \geq t} L_{n}\left(\tau_{k}\right)
$$

noting the fact that $(s-t)^{2} \leq s^{2}$ for $s \geq t \geq 0$. It is obvious that

$$
F_{n}(s) \leq 2 s^{2}|f(s, c)|, \quad L_{n}\left(\tau_{k}\right) \leq 2 \tau_{k}^{2}\left|f_{k}(c)\right| .
$$

From the definition of $G_{n}, L_{n}$ and conditions H2, H3, we obtain

$$
\lim _{n \rightarrow+\infty} F_{n}(s)=0, \lim _{n \rightarrow \infty} L_{n}\left(\tau_{k}\right)=0 .
$$

From (9), (10), (11) and the Lebesgue convergence theorem, we have

$$
\lim _{n \rightarrow \infty}\left\|S y_{n}-S y\right\|=0
$$

which means that $S$ is continuous.

(c) To show $S Y$ is precompact, we see that $(S y)(t), y \in Y$, is uniformly bounded. Now we will prove that $S Y$ is an equicontinuous family of functions on $R+$.

For $y \in Y$ and $t_{2}>t_{1}>0$, we have

$$
\begin{gathered}
\left|(S y)\left(t_{2}\right)-(S y)\left(t_{1}\right)\right| \\
\leq \frac{1}{2}\left|\int_{t_{2}}^{\infty}\left(s-t_{2}\right)^{2} f(s, y(s)) d s-\int_{t_{1}}^{\infty}\left(s-t_{1}\right)^{2} f(s, y(s)) d s\right| \\
+\frac{1}{2}\left|\sum_{\tau_{k} \geq t_{2}}\left(\tau_{k}-t_{2}\right)^{2} f_{k}\left(y\left(\tau_{k}\right)\right)-\sum_{\tau_{k} \geq t}\left(\tau_{k}-t_{1}\right)^{2} f_{k}\left(y\left(\tau_{k}\right)\right)\right| \\
\leq \int_{t_{1}}^{\infty} s^{2}|f(s, y(s))| d s+\sum_{\tau_{k} \geq t_{1}} \tau_{k}^{2}\left|f_{k}\left(y\left(\tau_{k}\right)\right)\right| \\
\leq \int_{t_{1}}^{\infty} s^{2}|f(s, c)| d s+\sum_{\tau_{k} \geq t_{1}} \tau_{k}^{2}\left|f_{k}(c)\right| .
\end{gathered}
$$

For any given $\varepsilon>0$, there exists $T_{1}>T$ such that 


$$
\int_{T_{1}}^{\infty} s^{2}|f(s, c)| d s+\sum_{\tau_{k} \geq T_{1}} \tau_{k}^{2}\left|f_{k}(c)\right|<\varepsilon .
$$

Hence, for any $t_{2}>t_{1} \geq T_{1}$, from (8), we have $\left|(S y)\left(t_{2}\right)-(S y)\left(t_{1}\right)\right|<\varepsilon$ for all $y \in Y$.

For $T \leq t_{1}<t_{2} \leq T_{1}$,

$$
\begin{aligned}
& \left|(S y)\left(t_{2}\right)-(S y)\left(t_{1}\right)\right| \\
& \leq \frac{1}{2}\left|\int_{t_{2}}^{\infty}\left(s-t_{2}\right)^{2} f(s, y(s)) d s-\int_{t_{1}}^{\infty}\left(s-t_{1}\right)^{2} f(s, y(s)) d s\right| \\
& +\frac{1}{2}\left|\sum_{\tau_{k} \geq t_{2}}\left(\tau_{k}-t_{2}\right)^{2} f_{k}\left(y\left(\tau_{k}\right)\right)-\sum_{\tau_{k} \geq t_{1}}\left(\tau_{k}-t_{1}\right)^{2} f_{k}\left(y\left(\tau_{k}\right)\right)\right| \\
& =\frac{1}{2}\left|\int_{t_{2}}^{\infty}\left(s-t_{2}\right)^{2} f(s, y(s)) d s-\int_{t_{1}}^{t_{2}}\left(s-t_{1}\right)^{2} f(s, y(s)) d s-\int_{t_{1}}^{\infty}\left(s-t_{1}\right)^{2} f(s, y(s)) d s\right| \\
& +\frac{1}{2}\left|\sum_{\tau_{k} \geq t_{2}}\left(\tau_{k}-t_{2}\right)^{2} f_{k}\left(y\left(\tau_{k}\right)\right)-\sum_{t_{1} \leq \tau_{k}<t_{2}}\left(\tau_{k}-t_{1}\right)^{2} f_{k}\left(y\left(\tau_{k}\right)\right)-\sum_{t_{2} \leq \tau_{k}}\left(\tau_{k}-t_{1}\right)^{2} f_{k}\left(y\left(\tau_{k}\right)\right)\right| \\
& \leq \frac{1}{2}\left|\int_{t_{2}}^{\infty}\left[\left(s-t_{2}\right)^{2}-\left(s-t_{1}\right)^{2}\right] f(s, y(s)) d s\right|+\frac{1}{2} \int_{t_{1}}^{t_{2}}\left(s-t_{1}\right)^{2}|f(s, y(s))| d s \\
& +\frac{1}{2}\left|\sum_{\tau_{k} \geq t_{2}}\left[\left(\tau_{k}-t_{2}\right)^{2}-\left(\tau_{k}-t_{1}\right)^{2}\right] f_{k}\left(y\left(\tau_{k}\right)\right)\right|+\sum_{t_{1} \leq \tau_{k} \leq t_{2}}\left(\tau_{k}-t_{1}\right)^{2}\left|f_{k}\left(y\left(\tau_{k}\right)\right)\right| \\
& \leq\left|t_{2}-t_{1}\right|\left[\int_{t_{2}}^{\infty} s^{2}|f(s, c)|\left|d s+\sum_{\tau_{k} \geq t_{2}} \tau_{k}^{2}\right| f_{k}(c) \mid\right] \\
& +\int_{t_{1}}^{t_{2}} s^{2}|f(s, c)| d s+\sum_{t_{1} \leq \tau_{k}<t_{2}} \tau_{k}^{2}\left|f_{k}(c)\right| \\
& \leq M\left|t_{2}-t_{1}\right|+\int_{t_{1}}^{t_{2}} s^{2}|f(s, c)| d s+\sum_{t_{1} \leq \tau_{k}<t_{2}} \tau_{k}^{2}\left|f_{k}(c)\right| .
\end{aligned}
$$

Hence, for any given $\varepsilon>0$, there exists a $\delta>0$ such that

$$
\left|(S y)\left(t_{2}\right)-(S y)\left(t_{1}\right)\right|<\varepsilon, \quad\left|t_{2}-t_{1}\right|<\delta,
$$

for all $y \in Y$.

That is, the interval $[T,+\infty)$ can be divided into a finite number of subintervals 
on which every $(S y)(t), y \in Y$, has oscillation less than $\varepsilon$.

Therefore, $S Y$ is an equicontinuous family on $[T, \infty)$.

We prove that the set $S Y$ is equiconvergent to $\infty$.

The definition of the operator $S$ implies

$$
\begin{gathered}
\left|(S y)(t)-\frac{c}{2}\right| \\
\leq \frac{1}{2}\left[\int_{t}^{\infty} s^{2}|f(s, y(s))| d s+\sum_{\tau_{k} \geq t} \tau_{k}^{2}\left|f_{k}\left(y\left(\tau_{k}\right)\right)\right|\right] \\
\leq \frac{1}{2}\left[\int_{t}^{\infty} s^{2}|f(s, c)| d s+\sum_{\tau_{k} \geq t} \tau_{k}^{2}\left|f_{k}(c)\right|\right]
\end{gathered}
$$

Hence, for any given $\varepsilon>0$, there exists a point $T_{\varepsilon}>T$ such that

$$
\int_{T_{\varepsilon}}^{\infty} s^{2}|f(s, c)| d s+\sum_{\tau_{k} \geq T_{\varepsilon}} \tau_{k}^{2}\left|f_{k}(c)\right|<2 \varepsilon .
$$

From (12) and (13), for $t \geq T_{\varepsilon}$ we get $\left|(S y)(t)-\frac{c}{2}\right|<\varepsilon$ for all $y \in Y$. Therefore $S Y$ is equiconvergent at $\infty$. Lemma 1 implies that the set $S Y$ is relatively compact.

According to the Schauder fixed point theorem, there exists a $y \in Y$ such that $y=S Y$. This $y$ is a bounded nonoscillatory solution of (1). The proof is complete.

Theorem 3: Assume that conditions H1-H3 hold. Then condition

$$
\int_{0}^{\infty} t^{2}|f(t, c)| d t+\sum_{k=1}^{\infty} \tau_{k}^{2}\left|f_{k}(c)\right|<+\infty
$$

for some constant $c \neq 0$ is necessary and sufficient for the existence of a bounded oscillatory solution $y$ such that $\lim _{t \rightarrow \infty} y(t)=d, d \neq 0$.

\section{References}

[1] Gopalsamy, K. and Zhang, B.G., On delay differential equations with impulses, J. Math. Anal. Appl. 139:1 (1989), 110-122.

[2] Györi, I. and Ladas, G., Oscillation Theory of Delay Differential Equations with Applications, Clarendon Press, Oxford 1991.

[3] Ladde, G.S., Lakshmikantham, V. and Zhang, B.G., Oscillation Theory of Differential Equations with Deviating Arguments, Pure and Applied Mathematics 110, Marcel Dekker 1987.

[4] Philos, Ch.G., Oscillatory and asymptotic behavior of the bounded solutions of differential equations with deviating arguments, Hiroshima Math. J. 8:1 (1978), 31-48.

[5] Shevelo, V.N., Oscillations of Solutions of Differential Equations with Deviating Arguments, Naukova Dumka, Kiev 1978. (In Russian) 


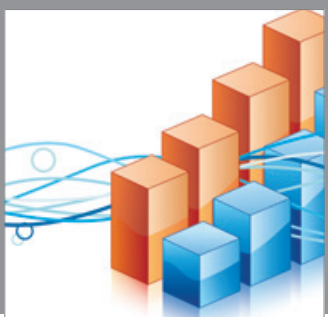

Advances in

Operations Research

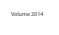

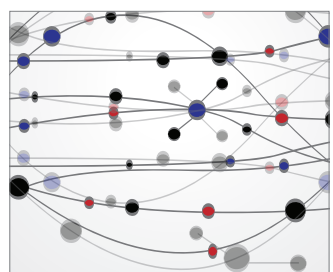

\section{The Scientific} World Journal
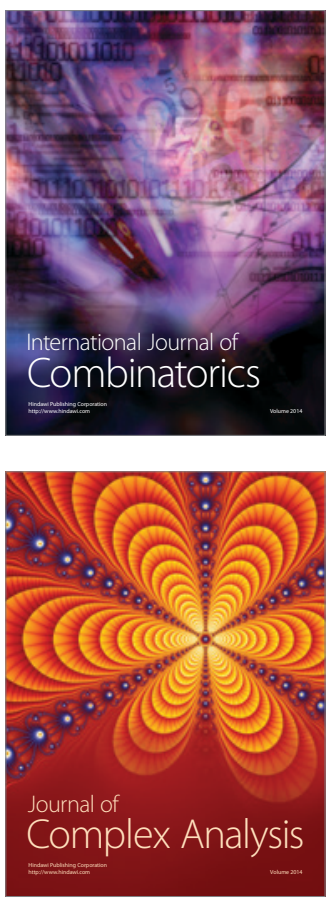

International Journal of

Mathematics and

Mathematical

Sciences
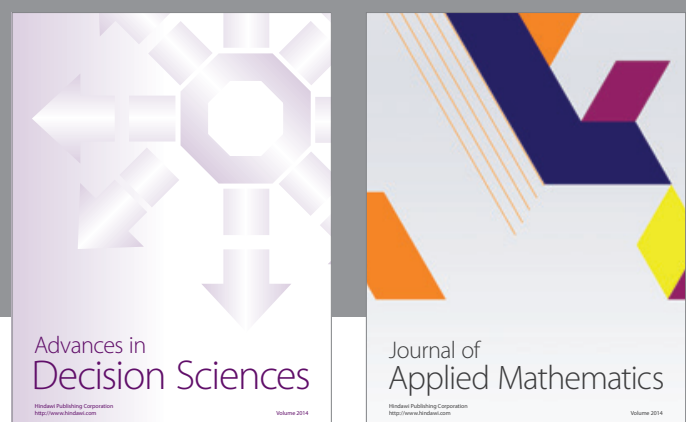

Journal of

Applied Mathematics
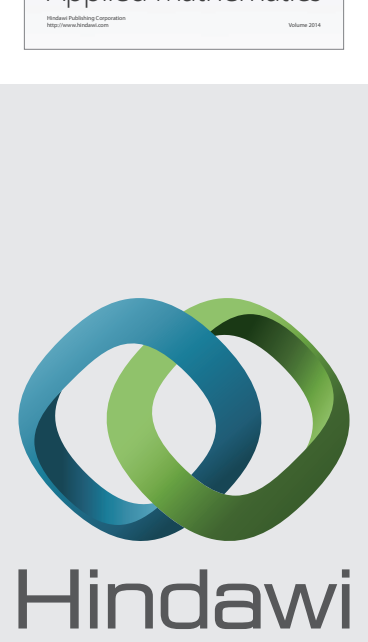

Submit your manuscripts at http://www.hindawi.com
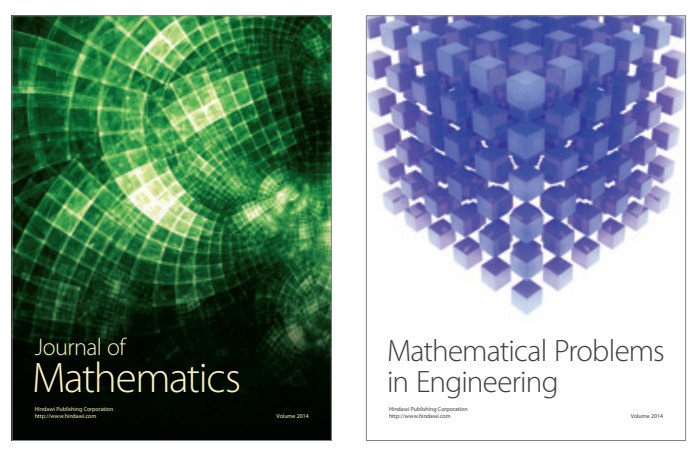

Mathematical Problems in Engineering
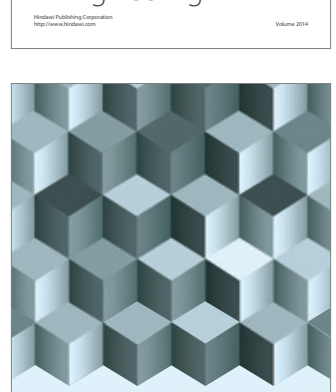

Journal of

Function Spaces
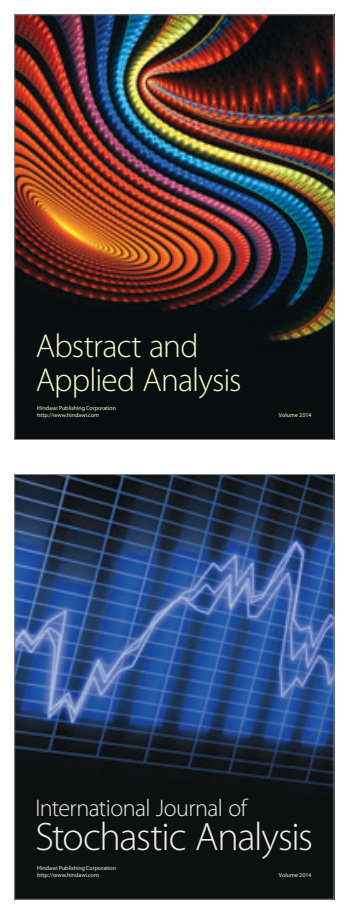

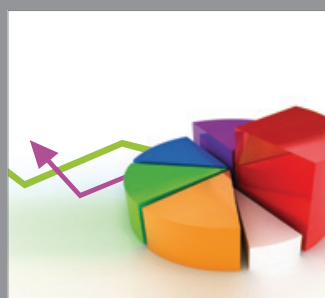

ournal of

Probability and Statistics

Promensencen
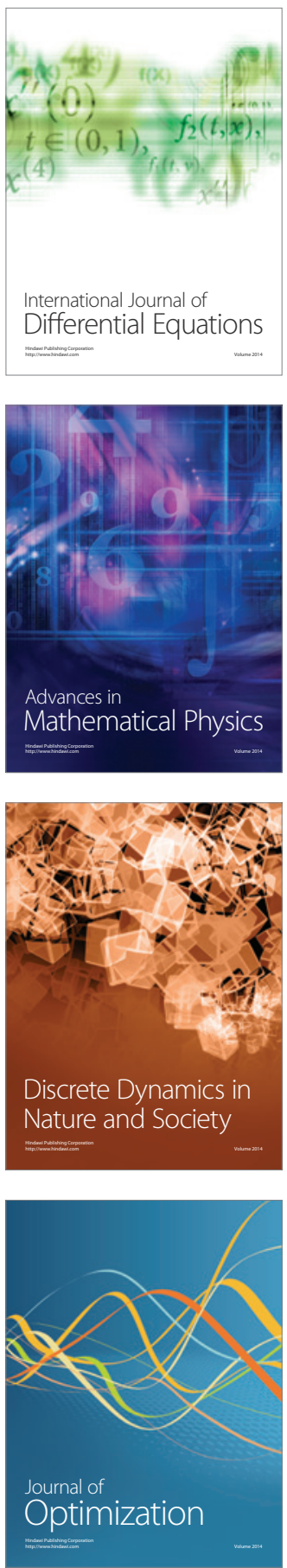